\title{
The Portuguese urban system: an opposition between its hierarchical organization in cyberspace vs. physical space
}

\begin{abstract}
The advent of computer and information technologies during the past decades, and their widespread use, is radically transforming the dynamics of communication and our understanding of spatial relationships. This empirical analysis intends to question how this kind of technological changes affect past trends in urban and regional development, specifically if they have enough potential to generate substantial adjustments on traditional Portuguese urban hierarchy, or instead, if it is an unexpected contribute to reinforce territorial disintegration tendencies. We analyse some geographic measurements of internet ownership, especially the density of .pt domains and the presence of each Portuguese city in the most used web search engines, in order to verify the plasticity of space throughout Portugal by the growing use of ICT's, and the ways it has been stretched or compressed. The findings of this study suggest that the role of ICTs in the struggle against spatial inequalities in Portugal is less relevant than expected.
\end{abstract}

Keywords: internet, cyberspace, urban hierarchy, Portugal

\section{Introduction}

This article intends to contribute to the discussion regarding which Portuguese cities are best positioned on the space of flows ${ }^{1}$ that characterizes the information societies. This abstract space needs nodal points to organize the networks of exchange of capital, decisions and information flows, and if we assume that the Internet will have a major impact for citizens and firms to exert their influence globally, we should ask where in Portugal can we find the individuals/bodies/activities with capacity to explore more efficiently the use of this telecommunication system.

Despite the rapid growth of Internet in recent years, and the gradual understanding of its implications, there is a lack of knowledge about the geographic diffusion of this advanced telecommunication service and the corresponding implications in urban development. Like Moss and Townsend (2000a, p. 39) said, "the urban planning 
profession has remained blissfully ignorant of both the negative implications and potential benefits of new telecommunications technologies".

The few academic empirical researches that have been examining the geography of Internet are mainly focused on four types of measurement. The most widely used geographic measurement of Internet activity is the number of hosts, or computers connected to the Internet, but this type of measurement is criticized because it simply focuses on a technological availability. Moss and Townsend (2000b) consider that the analysis of the number of domain names registered by geographic area is a much better technique, as it represents a social construct, since it corresponds to the entities with websites. The density of IP addresses is another indicator commonly used, despite its limitation since the geographical location of the entity owner of the IP address may not necessarily be the same where the Internet-linked computers are located. The geographic structure of backbone networks offers a fourth aspect that can be measured as an indicator of new flows of communications relationships emerging between places, and its configuration can be seen as a market response to localized demand for longdistance data transport services.

In this article we will try to contribute to the understanding of the geography of the Internet, and more specifically of the variations in diffusion rates among Portuguese municipalities and cities. To achieve this goal we will test two types of measurements: the addresses of all registration. $\mathrm{pt}^{2}$ domain names, and the number of links to each one of all Portuguese cities provided by the most used web search engines: in Portugal (www.sapo.pt) and in worldwide (www.google.com). This last type of measurement is one additional contribution trying to deal with the need for experimentation with new empirical techniques and measurements to study the relationships between Internet and urban systems.

\section{Telematics implications for urban development}

In recent years, attention is increasingly being drawn to the investigation of how the economic, social and cultural aspects of cities interact with the proliferation of advanced computer-based telecommunications networks. Therefore, the co-evolution of cities and tele-mediated interactions is progressively emphasised in academic debates concerning 
the future of cities. As a consequence of the recognized influence of ICTs on the changes in urban structures and everyday urban life, we can point out three main perspectives that have been dominating the discussion about how recent advances in telematics will affect urban development:

- the digital city perspective;

- the global city perspective;

- $\quad$ and the urban dissolution perspective.

The digital city perspective can be presented as a means of creatively integrating telecommunications into urban policy and planning practices and strategies, in order to develop more inclusive and sustainable urban futures. The emergence of this concept can be seen as an effort to develop and use advanced telematics solutions for the improvement of urban dynamics, with different goals and meanings according to the selected organizational format, the technical solution adopted, and especially the agents and organizations involved, with their different aims and resources.

Although there is no common definition regarding the digital cities concept, since its goals, architectures and motivations are expected to continue to evolve together with the computer and communication technologies, we can present two different typologies in an attempt to systematise its various meanings.

The first meaning is related to the representation of cities as community networks, essentially based on the WWW. In this model, a digital city is seen as a local information service, working as a platform for community networks, in which people and institutions are expected to interact. In this typology, we can consider the digital cities as local information services for the citizens' everyday life, as well as for visitors (in this case we are dealing with websites as information centres, providing locally relevant news, as well as information on entertainment and commerce, tourist attractions, hotels reservations, useful addresses and other related items).

The second meaning of the concept refers to virtual communities and virtual environments based on urban metaphors, typically including 3D technologies, in some cases designed to allow citizens to participate in the ongoing urban planning process. In this case, a digital city can be seen as a platform for communities of interest, with the purpose of working not only in the geographical space but also in the information space, where users can interact, sharing knowledge, experience and mutual interests. 
In recent years, following one of these meanings, various digital cities have been created around the world by using ICTs creatively, with quite different goals and motivations. On the one hand, we have very resourceful initiatives, developing advanced cutting edge technologies, whereas others are known as low budget initiatives, using established and well-known technologies. On the other hand, some were meant to be a sustainable part of the local infrastructures, using ICTs as instruments for supporting long term urban development strategies, whereas others are merely temporary experiments for demonstration purposes.

The global city perspective underlines the dominant role of a selected group of 'global cities', such as New York, London and Tokyo, as nodes in the global flows of information that characterizes the network society, playing a particular and unique role in the process of global economic restructuring. This theoretical framework argues that ICT's provide the means for those cities to perform some control and command functions in order to coordinate networks for the production and distribution of information, money and power. Townsend (2000) criticizes this theory and argues that the growth of the Internet since the mid 90's represents a significant change in the technological framework and is a challenge to the established order, offering an opportunity to reshape urban hierarchies within the global economy.

The urban dissolution perspective, founded on a long tradition of utopian thoughts, has recently been revived since the Internet began growing steadily. These prophetic views argue that cheap and sophisticated telecommunications abolish the need for faceto-face relationships, promoting a radical decentralization of population and production, with an expected obsolescence of cities. But Townsend (2000) criticizes this theory, since his empirical investigation suggests an overwhelmingly metropolitan dominance of Internet activity and infrastructure, which are new signals of continued agglomeration tendencies.

In Townsend's view, either the global city or the urban dissolution perspectives involves a great deal of speculation and little empirical evidence to support these claims. He proposes a new theoretical framework based on empirical findings that describes the geographic diffusion of Internet activity and infrastructure in the United States between 1969 and 1999, which allows him to say that a new network of urban spaces is emerging, structured by many cities that have been able to successfully market their 
own services on a global scale. "Rather than a handful of regions dominating global finance and culture, a constellation of highly specialized regions now interact in a globally telemediated economy" (Townsend, 2000, p.43)

This proposal of urban hierarchy rearrangement is based on the measurement and analysis of the geographic structure of Internet communications, taking into account its huge potential to promote remarkable shifts in economic and cultural geographies. He concludes that although New York and Los Angels represent the USA's two largest clusters of Internet activity by the measure of domain names ${ }^{3}$, in terms of density of adoption there are several metropolitan areas that outpace the traditional global cities.

"New York and Los Angels clearly contain the largest clusters of Internet activity. (...) Chicago, which is typically considered a global city in the academic literature, ranks closer to Boston than to either New York and Los Angels. Furthermore, there are a number of medium-sized metropolitan areas that top the list - including Boston, Seatle, Miami and Atlanta." (Townsend, 2001, p. 50-51)

Dodge and Shiode (2000) conducted another academic empirical research that examined the geography of Internet in a detailed geographic scale. In their exploratory analysis in the UK, using the density of IP addresses ${ }^{4}$, they concluded that the majority of the UK's Internet space is owned by commercial organizations, which are located in a few urban centres: Central London and its surrounding satellite towns (as expected); Nottingham (due to a large block of address space allocated to an ISP - Internet Service Provided- placed in Nothingham ${ }^{5}$ ) and Cambridge (with a high concentration of computing, research-oriented companies and the university).

The results of this study also contradict the urban dissolution perspective and underline that the Internet, rather than disintegrating cities and major metropolitan regions, will reinforce the comparative advantages that such regions have as centres for information production and transmission, which reflects the significance of face-to-face contact in business and social life in order to generate innovative individuals and firms.

But what have been the implications of the Internet widespread use in our understanding of the Portuguese spatial relationships? Are we facing a communication technology with enough potential to generate substantial adjustments on traditional 
Portuguese urban hierarchy, or instead, has it been unexpectedly contributing to reinforce territorial disintegration tendencies?

\section{The geography of .pt domain names}

The globally-interconnected set of computer networks, communicating via an agreed set of protocols, known as 'The Internet', is widely used by millions of individuals, firms and institutions every day, as a way to get into an invisible domain, popularly known as cyberspace, in order to seek information, market new services and products, and to establish communication within and between organizations. However, like Dodge and Shiode (2000) said, these invisible territories of the cyberspace do have a geography and, in an attempt to reveal its topography, we should analyse the spatial patterns of the ownership of Internet space.

In order to examine the geography of Internet in Portugal we need to find links between cyberspace and the Portuguese physical geography. A good way to achieve this goal is to determine the location of Portuguese institutions that recognize the importance of distributing information globally, about their activities and services, through the use of the Internet. Since the content of any type of domain name ${ }^{6}$ is globally accessible, we will begin by exploring the compulsory registration of all .pt domain names in one single institution -Foundation for National Scientific Computing(FNSC), which performs all the registration services for the geographic .pt domain.

Just before presenting the main results of our analysis, we should now clarify some limitations of this methodological approach. Firstly, we did not study all Portuguese domain names since we did not have access to CONE domains ${ }^{7}$ registrations done by the Portuguese institutions, and therefore these results represent only a part of the Portuguese Internet content providers. In addition, this spatial pattern may not necessarily indicate the location of the activities of production of Internet contents in Portugal, since those services can be provided remotely. Finally, Steineke (2000, p.13) states that we have to be attentive, since the practice of domain grabbing is sometimes responsible for high domain densities "when private speculators register intuitive or trademarked names with the hope of selling the rights to these names to others at a latter date". 
Although we did not have access to CONE domains registrations, according to the Anacom, which is the institution that regulates, supervises and represents the communications sector in Portugal, at the end of 2003 there were about 25 thousand domain names registered, and the pt domain, having more than $75 \%$ of all domains registered in Portugal, is definitely the more significant of all typologies (Figure 1). Therefore, studying the pt domain name will give us a good representative picture of the geography of Internet in Portugal.

Figure 1. Evolution of Portuguese internet domain names (1998-2003)

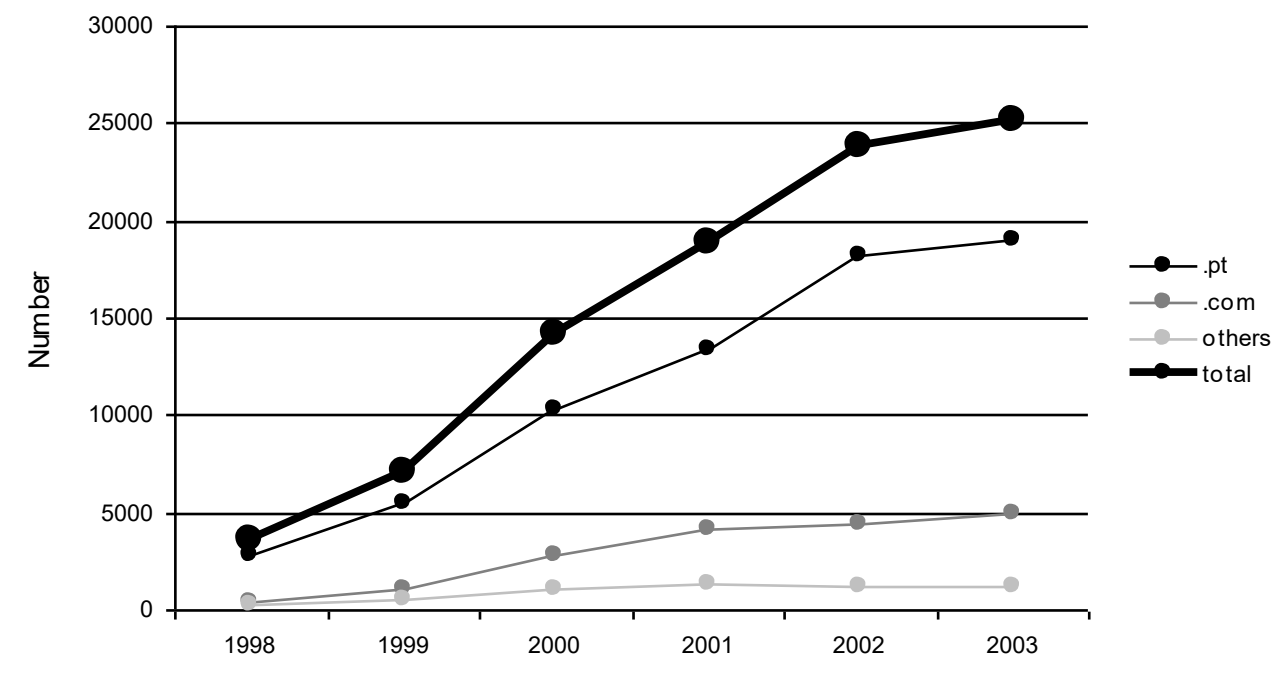

Data collected in: Anacom (2004)

FNCS began accepting registrations in 1991, but it was only at the beginning of 2001 that the .pt domains in Portuguese institutions became more common, with more than 3 thousand new domains every semester (Figure 2). Despite this recent acceleration, preceded by a decade of slow acceptance, we are still facing an emerging phenomena throughout Portugal concerning the diffusion of .pt domains. 
Figure 2. Number of .pt domain names registered by semester since 1995

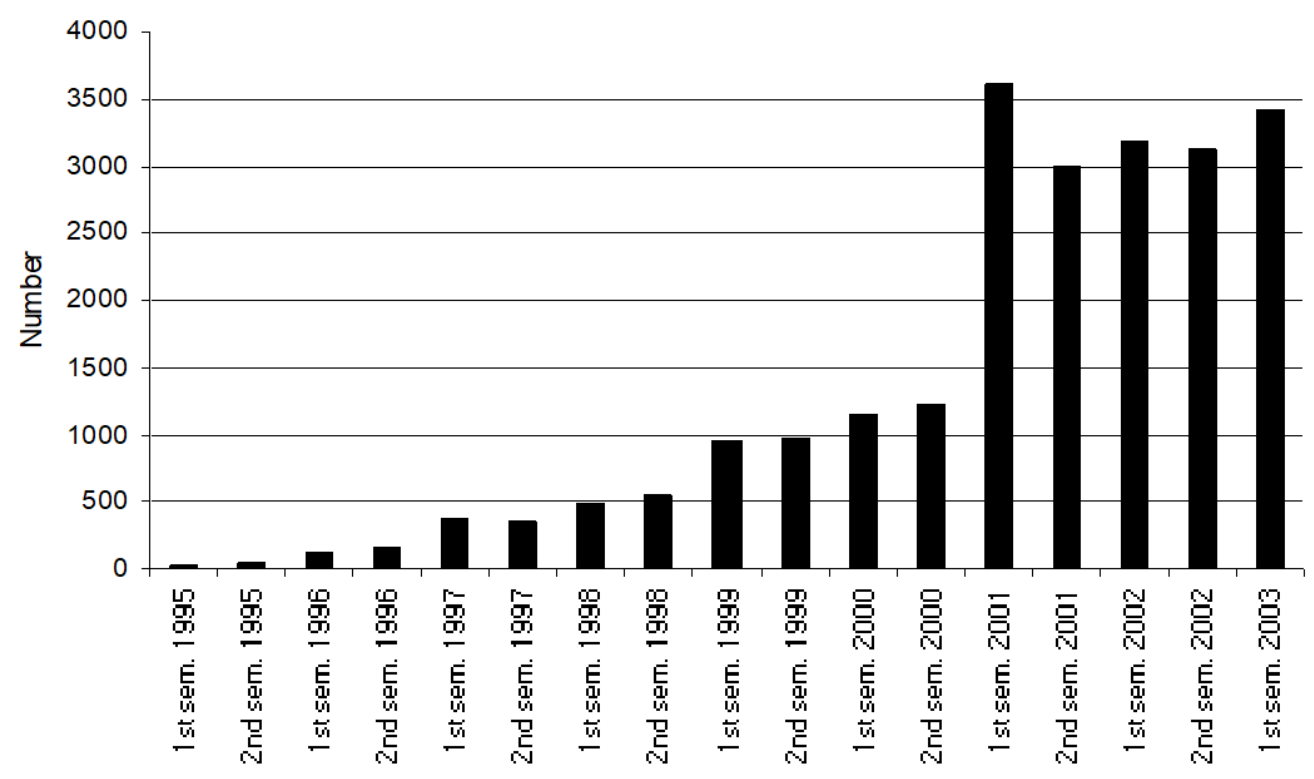

Data collected in: Foundation for National Scientific Computing (2003)

The administration of FNSC gave us the addresses of almost all entities with an Internet presence under de .pt top level domain ${ }^{8}$, which allowed us to patiently pinpoint a geographical location, by municipality, for the ownership of 24724 .pt domain names (using the zip codes of the mailing address details), which turned out to be extremely useful for discovering geographic variations in the Portuguese Internet ownership.

Surprisingly perhaps, there is not a correspondence between the number of .pt domains presented by Anacom and the number revealed by FNSC. This difference may probably be due to the fact that Anacom does not consider the total registrations but only the active domains. We should not forget that some of the registered institutions stop paying the amount that is required for the biennial maintenance of their domains.

A small part of these domains (around two thousand) belongs to foreign institutions, which for some reason felt it would be profitable to promote their objectives and services through the use of a website with a .pt domain. The six most represented countries are (starting with the most important): the USA, Switzerland, Spain, Germany, France and the United Kingdom. These are countries with strong relations with Portugal, not only as a direct result of sustained business links (apart from Switzerland, they are all part of the top 7 Portuguese import and export countries), but also as 
countries with the more significant Portuguese emigrant communities. On the one hand, we have the USA, France and Germany as a long date receivers for Portuguese emigration, on the other hand we have Switzerland, Spain and United Kingdom, which have been more recently chosen as destinations for Portuguese emigrants.

Considering only the .pt domains that belong to entities based in Portugal, and in order to understand the logic of the spatial diffusion process throughout the country, we have decided to analyse each of the .pt domains according to the semester in which they have been registered. However, more important than discovering the date of the first registration in each municipality, we decided to mark the semester in which a continuous registration began, which means that we marked the first semester of a set of registrations that continued up to the present date.

The pioneer municipality with a systematic register of .pt domains was the Portuguese capital Lisbon (1991), followed by Oeiras (1993) and Cascais (1995). All of these are located in the Lisbon Metropolitan Area, which is the Portuguese leading financial and administrative centre, concentrating a large and varied set of advanced services, including the head offices of the largest domestic and multinational companies.

The second diffusion centre was established at the end of 1995 in the north of Portugal, structured by a node around Porto (Porto, Vila Nova de Gaia and Matosinhos) and another one in Braga. The Porto Metropolitan Area and its surrounding municipalities form an almost continuous urban sprawl, generally known as city agglomeration, which comprises an extensive area, stretching north as far as Braga.

At a later stage, a new diffusion phase began (between 1996 and the beginning of 1998), which was characterized by the appearance of a set of other leading municipalities that correspond basically to the medium-sized cities that form the urban coastal system that have been growing between these two metropolitan systems (it is the case of Aveiro, Coimbra, Figueira da Foz and Leiria/Marinha Grande). In this phase, the urbanization of the coast of Algarve is also a relevant phenomenon in the appearance of new websites.

This diffusion process allows us to conclude that the conquest of cyberspace in Portugal began throughout the most urbanised municipalities, with a better economic situation and better served in terms of road accessibility. Moreover, as we will now 
demonstrate, there is still a definite correspondence between these pioneering municipalities and the Portuguese localities with the largest clusters of Internet activity at the present time.

In order to determine which are the Portuguese localities that nowadays participate more actively in the global flows of information, we decided to use the density of domain name registrations in relation to the local population, rather than to analyse the magnitude by municipalities since it would understandably reveal the most populated ones.

Looking at the spatial distribution of the domain density (number of .pt domain levels per 10000 inhabitants, by municipality) we can determine the most wired Portuguese localities in 2003 (Figure 3). If we consider only the municipalities with a density higher than the national average (22.pt domains per 10000 inhabitants) we may highlight their agglomeration into five main areas, all of them located in the Portuguese urban coastal system:

1) The north extension of the Porto agglomeration (Porto, Matosinhos, Maia, Santo Tirso and Braga), which has a significant demographic potential, a productive structure not so rich or diversified in terms of services, and a more traditional but strongly exportoriented industrial profile.

2) A group of municipalities gathered around Aveiro and Coimbra. Aveiro, thanks to its University and Research Centres, presents new areas for expansion and internationalization (telecommunications, electronics and informatics), and, together with its neighbouring municipalities (Águeda and Albergaria-a-Velha), has a dynamic production system based on ceramics and metallurgy. Coimbra polarizes an extended area of influence and its University, as well as a science complex linked to health care services, distinguishes the city and give it an international dimension.

3) Additionally, Leiria, Batalha, Ourém and Marinha Grande constitute a significant urban system and an internationalised industrial cluster linked to the plastics injection moulding industry.

4) The north section of the Lisbon Metropolitan Area (Lisbon, Loures, Amadora, Oeiras, Cascais, Sintra and Mafra) is intensively urbanized and is clearly characterized by a predominance of services for industry, financial services, as well as cultural and recreational activities. 


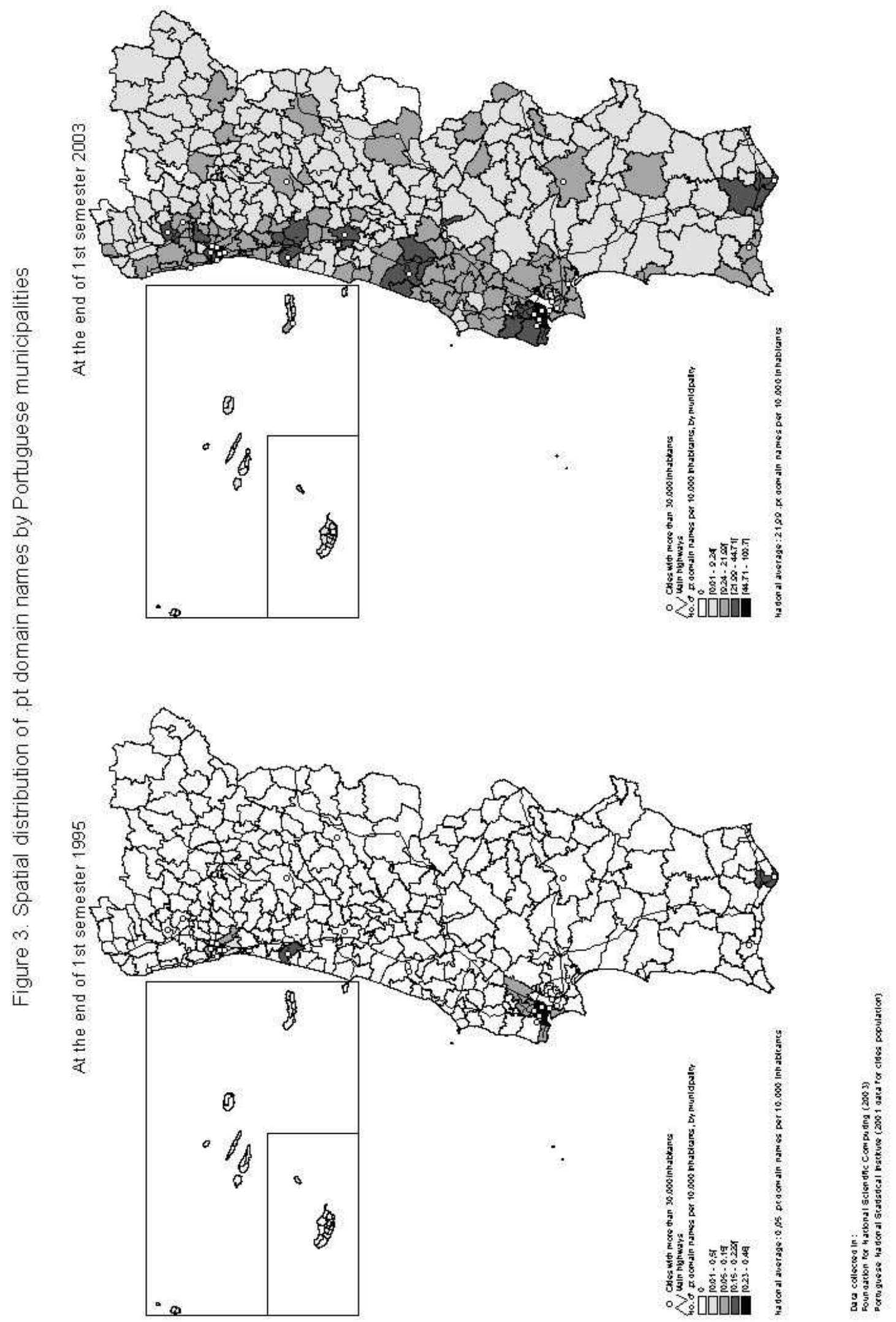


5) Finally, the urbanization of the south coast (especially in the municipalities of Faro and Loulé) is strongly linked to the Algarve potential as regards international tourism. Nowadays, many hotel reservations are made over the Internet, and the numerous hotels located in Algarve offer websites connected to several on-line booking agencies.

In conclusion, it is possible to say that there is a strong relationship between the geographic patterns of domain ownership in Portugal and the municipalities that include the biggest cities and that have a more competitive and internationalised economic profile. So far, these indications allow us to say that the use and appropriation of new telecommunications systems have been aggravating the territorial disintegration tendencies, instead of helping to overcome the traditional geographical barriers.

\section{The distribution of .pt domain names by Portuguese economic activities}

Surprisingly, while in the geographic analysis we found a clear correspondence between the most dynamic municipalities and the localities that nowadays participate more actively in the global flows of information, the economic analysis shows that there is some relevant discrepancies between the most prosperous Portuguese economic branches (in terms of number of firms) and those that realize the potential of owning Internet space (Table 1).

Table 1. Comparison between economic activities more represented in terms of firms and .pt domains

\begin{tabular}{|c|c|c|c|c|c|c|}
\hline & \multicolumn{3}{|c|}{.pt domain names (2003) } & \multicolumn{3}{|c|}{ Firms $(2000)$} \\
\hline & Number & $\%$ & Order & Number & $\%$ & Order \\
\hline Agriculture, hunting and forestry & 19 & 0,1 & & 10430 & 3,4 & \\
\hline Fishing & 2 & 0,0 & & 303 & 0,1 & \\
\hline Mining and quarrying & 107 & 0,6 & & 1154 & 0,4 & \\
\hline Manufacturing & 2922 & 16,9 & $3^{\circ}$ & 48404 & 15,5 & $2^{\circ}$ \\
\hline Electricity, gas and water supply & 62 & 0,4 & & 619 & 0,2 & \\
\hline Construction & 722 & 4,2 & & 39800 & 12,8 & $3^{\circ}$ \\
\hline $\begin{array}{l}\text { Wholesale and retail trade; repair of motor vehicles, } \\
\text { motorcycles and personal and household goods }\end{array}$ & 4609 & 26,6 & $2^{\circ}$ & 102490 & 32,9 & $1^{\mathrm{o}}$ \\
\hline Hotels and restaurants & 265 & 1,5 & & 33282 & 10,7 & $4^{\circ}$ \\
\hline Transport, storage and communication & 771 & 4,5 & $5^{\circ}$ & 11993 & 3,9 & \\
\hline Financial intermediation & 298 & 1,7 & & 7761 & 2,5 & \\
\hline Real estate, renting and business activities & 4810 & 27,8 & $1^{\mathrm{o}}$ & 27766 & 8,9 & $5^{\circ}$ \\
\hline $\begin{array}{l}\text { Public administration and defence; compulsory social } \\
\text { security }\end{array}$ & 481 & 2,8 & & 261 & 0,1 & \\
\hline Education & 387 & 2,2 & & 3494 & 1,1 & \\
\hline
\end{tabular}




\begin{tabular}{|c|c|c|c|c|c|}
\hline $\begin{array}{l}\text { Health and social work } \\
\text { Other community, social and personal service activities } \\
\text { Extra-territorial organizations and bodies }\end{array}$ & $\begin{array}{c}245 \\
1592 \\
14\end{array}$ & $\begin{array}{l}1,4 \\
9,2 \\
0,1\end{array}$ & $4^{o}$ & $\begin{array}{c}11264 \\
12292 \\
4\end{array}$ & $\begin{array}{l}3,6 \\
3,9 \\
0,0\end{array}$ \\
\hline $\begin{array}{l}\text { TOTAL } \\
\text { Data collected in: } \\
\text { Foundation for National Scientific Computing, } 2003\end{array}$ & 17306 & 100,0 & & 311317 & 100,0 \\
\hline
\end{tabular}

For example, the economic branch concerning real estate, renting and business activities includes only $8.9 \%$ of all Portuguese firms, but, nevertheless, it is the economic branch with a more significant presence in the cyberspace, since these activities represent almost $28 \%$ of all Portuguese economic activities with .pt domains.

Another example is the construction sector, the third biggest economic sector in Portugal. This sector includes $12.8 \%$ of all firms, but it has only $4.2 \%$ of all .pt domain names, confirming that some types of private institution do not recognize the importance of globally distributing information regarding their activities and services, through the use of the Internet.

Another important conclusion resulting from this economic analysis has to do with the fact that the influence of Internet on regional development is in some cases a clear contribution to reinforce the tendency to territorial disparities, promoting geographic inequalities throughout the country even more pronounced than in real space. For the three economic branches with most pt domains (Tables 2, 3 and 4), we can conclude that the institutions with Internet websites are much more spatial concentrated comparing with their physical locations, which means that electronic communications reinforce existing patterns of physical communication rather than acting as equilibrium forces.

For instance, Lisbon concentrates $19.9 \%$ of all firms in the real estate, renting and business activities, but if we analyse the spatial distribution of all these activities with .pt domains we will observe a much higher agglomeration in Lisbon, with almost 34\% of all these websites (Table 2). In this economic sector, with the exception of Braga, the others nine municipalities with more pt domains are located in the two Portuguese metropolitan areas. A careful analysis allowed us to conclude that in a total of 308 Portuguese municipalities, just three of them (Lisbon, Porto and Oeiras) include $50 \%$ of all these Portuguese firms with .pt domains. 
Table 2. Top 10 municipalities with more .pt domains in the economic branch concerning real estate, renting and business activities

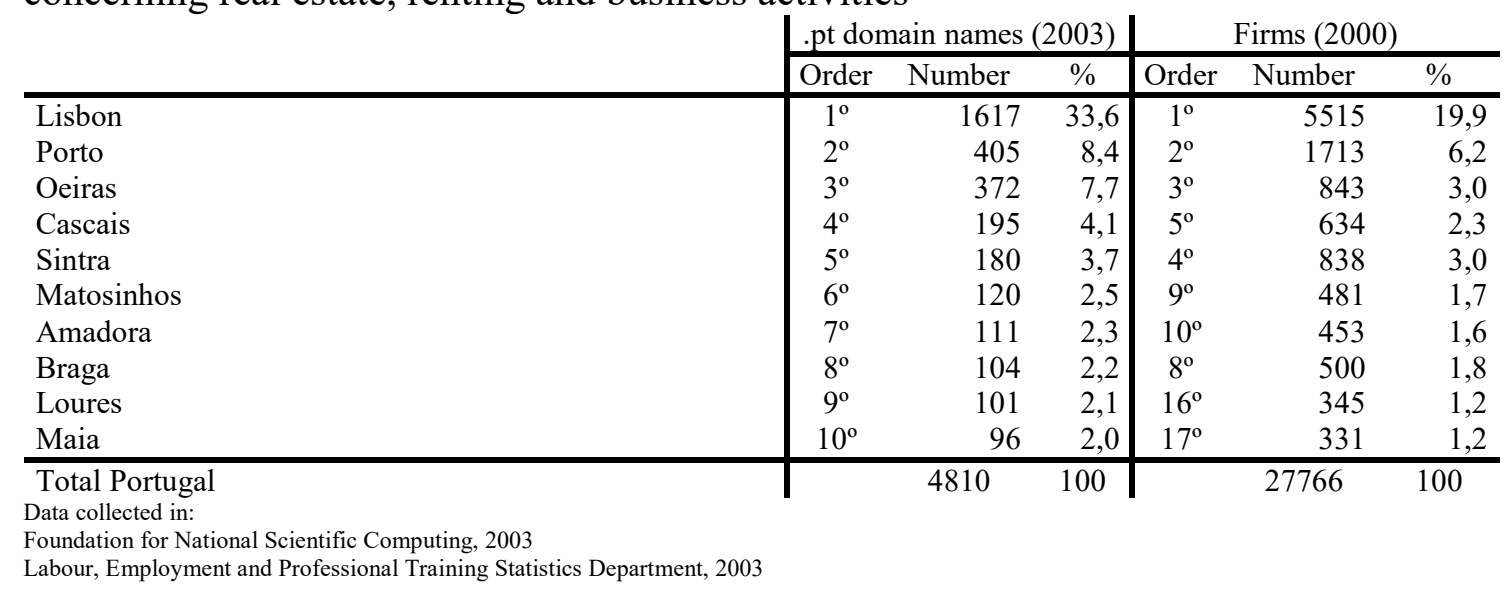

Concerning manufacturing activities, we can highlight the position of Oeiras (Table $3)$. Despite its $38^{\text {th }}$ place, Oeiras is the fourth Portuguese municipality in terms of the number of manufacturing activities with .pt domains. On the contrary, Guimarães is the locality where the higher number of manufacturing firms is based, however those activities are not so well represented in the global flows of information.

Table 3. Top 10 municipalities with more .pt domains in the economic branch concerning manufacturing activities

\begin{tabular}{l|crr|rrr} 
& \multicolumn{3}{c}{.pt domain names $(2003)$} & \multicolumn{3}{c}{ Firms $(2000)$} \\
\cline { 2 - 7 } & Order & Number & \multicolumn{1}{c}{$\%$} & Order & Number & $\%$ \\
\hline Lisbon & $1^{\circ}$ & 329 & 11,3 & $3^{\circ}$ & 1787 & 3,7 \\
Sintra & $2^{\circ}$ & 135 & 4,6 & $6^{\circ}$ & 1373 & 2,8 \\
Porto & $3^{\circ}$ & 118 & 4,0 & $10^{\circ}$ & 1081 & 2,2 \\
Oeiras & $4^{\circ}$ & 108 & 3,7 & $38^{\circ}$ & 336 & 0,7 \\
Vila Nova de Gaia & $5^{\circ}$ & 102 & 3,5 & $5^{\circ}$ & 1383 & 2,9 \\
Maia & $6^{\circ}$ & 83 & 2,8 & $19^{\circ}$ & 635 & 1,3 \\
Guimarães & $7^{\circ}$ & 80 & 2,7 & $1^{\circ}$ & 2021 & 4,2 \\
Loures & $8^{\circ}$ & 79 & 2,7 & $17^{\circ}$ & 643 & 1,3 \\
Águeda & $9^{\circ}$ & 78 & 2,7 & $23^{\circ}$ & 541 & 1,1 \\
Santa Maria da Feira & $10^{\circ}$ & 73 & 2,5 & $4^{\circ}$ & 1551 & 3,2 \\
\hline Total Portugal & & 2922 & 100 & & 48404 & 100
\end{tabular}

Foundation for National Scientific Computing, 2003

Labour, Employment and Professional Training Statistics Department, 2003

These figures allow us to say that the economic geography clearly reveals different patterns in physical space or in cyberspace. While it is clear that the Internet may contribute significantly to reinforce territorial dichotomies, the truth is that in some cases there are localities in which less stimulating economic sectors are exploring much more efficiently the potential offered by information and communication technologies. 


\section{Presence of Portuguese cities in the most relevant web search engines (www.google.com and www.sapo.pt)}

Apart from knowing where the Portuguese entities with websites are concentrated, we think that in the space of flows we should especially explore the global accessibility of the contents of these websites. To measure the accessibility values we will use the number of results found on web search engines, as this is the most common way to find information on the Internet. In cyberspace, more important than owning a website is to make its contents easily available, and we believe that the Portuguese localities with the highest number of websites with good accessibility are well positioned in the so called information highways.

In the most commonly used Portuguese web search engine (www.sapo.pt), as well as the most popular web search engine worldwide (www.google.com) we did 134 searches, one for each Portuguese city, thus obtaining the amount of web results for each city. We decided to take all the results into account, even with websites including several links, as we believe this fact indicates a better connectivity to the global flows of information. Moreover, more important than the magnitude of the web results for each city is their relative importance when compared with each other.

The results obtained include all kinds of domain names, and are not limited to .pt domains or institutional contents, which could prove to be more effective for a characterization of ownership of Internet space in Portugal.

The main limitation of this methodology has to do with the possibility of finding results that are not related to the city. This happens when we have other localities in other countries with the same name (for instance Barcelos is also a locality in Brazil), or when the designation of a city is also a surname (for instance Gouveia or Fátima), or even when we have a polysemic word in the city's name (Viana do Castelo with the word castelo, which means castle, or Ponte de Sôr with the word ponte, which means bridge). In order to find a solution to this problem, we checked each one of the results obtained in www.sapo.pt and eliminated the results that have no relation with the city. However, this method was not as effective with all the results obtained in www.google.com, because the number of results was significantly higher. For this 
reason, we chose to check only a sequence of 50 results for each city and make an extrapolation of the useless results. As a consequence, we believe that the analysis we carried out as regards the presence of Portuguese cities in the most used national web search engine (www.sapo.pt) is more trustworthy.

Finally, it is important to note that there are other methods to find information on the Internet. We must not forget the importance of links between websites for Internet navigation. Furthermore, in our search we did not get all websites belonging to entities or persons located in Portuguese cities, but only the ones that contain information about the physical location of the entity or the person that owns that website.

We believe these results are more easily understandable if we look at three maps, one of them representing Portuguese cities according to their amount of population and two others picturing the presence of Portuguese cities in the two web search engines that were analysed (Figure 4). To make all three maps comparable we decided to apply the same graduated symbol in the legend, and for all of them we used the natural breaks classification method to obtain 4 classes. The symbol of each class appears in a different colour to facilitate the comparison between them. We can draw three main conclusions from this examination.

Generally, the relative position in the urban hierarchy stays the same in cyberspace, that is, the more densely populated cities usually have a more significant presence on the Internet. But a more detailed examination allows us to infer that the use of these new telecommunications services generate considerable adjustments on the traditional Portuguese urban hierarchy. The more obvious adjustment is the absence in cyberspace of the biggest cities of the suburban rings of Lisbon and Porto.

In the www.sapo.pt, with the exception of Setúbal, the two metropolitan areas are surrounded by cities with fewer connections to the information highways, which can be directly associated with the urbanization processes in these suburban rings. The Lisbon metropolis is strongly centripetal receiving everyday thousands of people that live in peripheral municipalities (cities with a marked residence profile). In the Porto metropolis, we find some peripheral urban centres trying to increase their institutional dimension and autonomy, the role of these new centralities can already be seen with the cities of Matosinhos and Espinho being distinguished in www.google.com. 


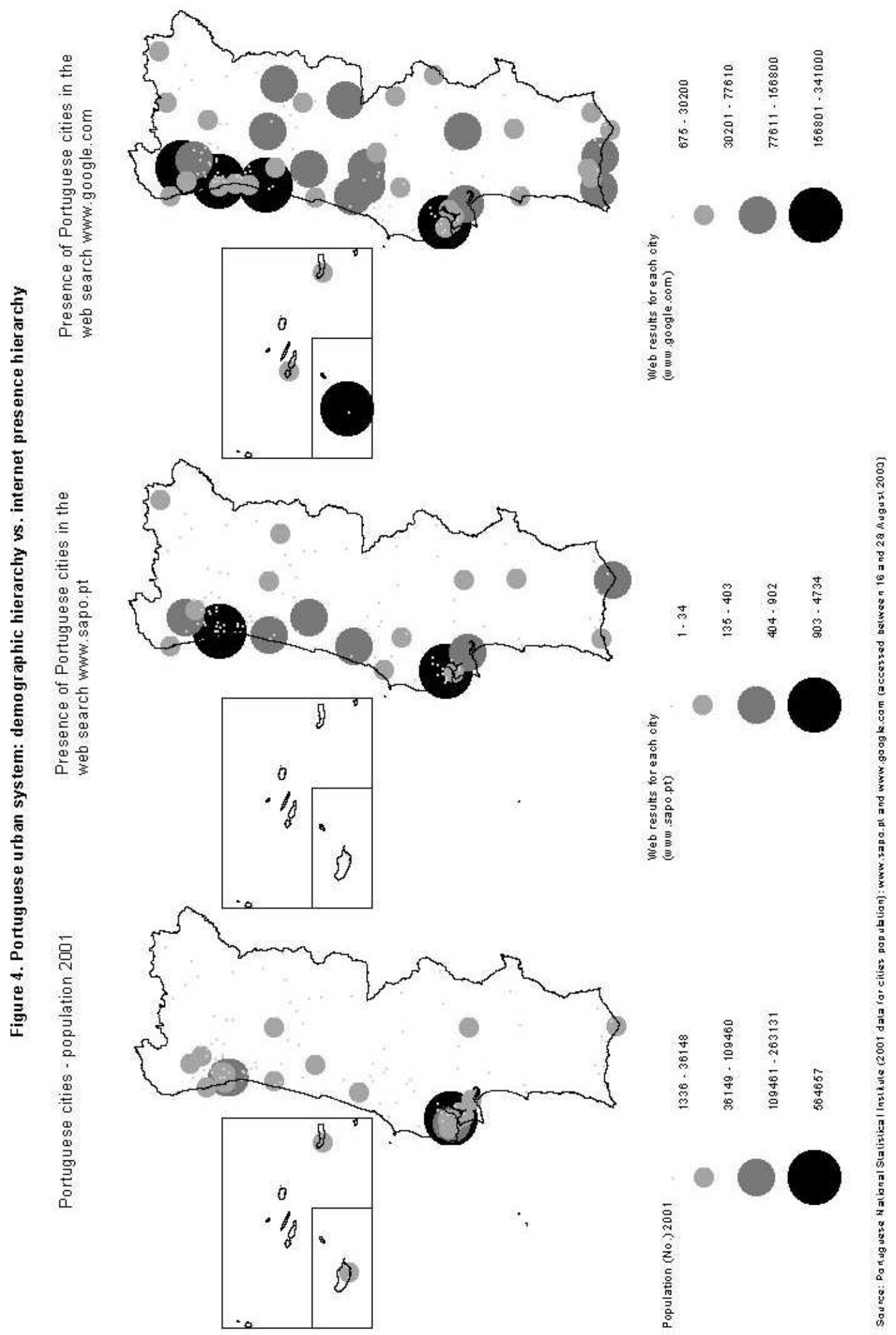


Another important conclusion is related to different types of hierarchical relationships between cities in the demographic and the Internet presence hierarchy, especially marked by an evident decrease of disparities. If we analyse the Portuguese cities according to their population, we find a short number of cities in the highest classes, since Lisbon, with a population of 565 thousand inhabitants, is the only city in the top class. In the second class we have only three cities, two of them located in the Porto metropolis (Porto and Vila Nova de Gaia) and the other one in the Lisbon metropolis (Amadora). However, we have a much more balanced hierarchy as regards the presence of Portuguese cities on the Internet, which is clear in www.sapo.pt and even more evident in www.google.com. Porto is, just after Lisbon, on the top class in www.sapo.pt, as well as Braga, Aveiro and Funchal in www.google.com. On the second class we have, in www.sapo.pt, five relevant cities outside the two metropolis (Braga, Aveiro, Coimbra, Leiria and Funchal), and www.google.com includes 11 cities, some of them outside the urban coast (Viseu, Guarda, Castelo Branco and Évora).

In brief, we have attested and confirmed the importance of big cities as the main nodal points as regards information exchange. However, there are some indicators suggesting that there can be a more balanced urban hierarchy concerning the general accessibility of the web contents provided in these cities.

\section{The Portuguese urban system: demographic hierarchy vs. internet presence hierarchy}

To finalise this analysis we decided to present the Portuguese cities with more than 30 thousand inhabitants crossing the results obtained with both methodologies: the spatial distribution of .pt domains and the results achieved on the web search engines. However, we chose to use only the results obtained in www.sapo.pt backed by three main reasons. Firstly, as well as the results of this search engine are more restricted, the analysis of domain names is also limited to a part of the Portuguese ownership of Internet space (only the .pt top level domain). In addition, as we explained before, the results obtained in www.sapo.pt are more trustworthy since we extrapolated the useless results presented by www.google.com. Finally, we calculated the correlation coefficient 
between the .pt registers located on the municipalities with cities and the web results for each city, and achieved a stronger correlation with the www.sapo.pt results (correlation coefficient 0.919) in comparison with the www.google results (correlation coefficient $0.573)$.

This combined analysis allowed to determine seven cities presenting a total of .pt domains and web results higher than the national averages, namely: Lisbon, Porto, Braga, Coimbra, Setúbal, Aveiro and Leiria (Figure 5).

Analysing the opposition between demographic hierarchy and Internet presence hierarchy it is important to emphasize that cities located in the suburban rings of the two Portuguese metropolitan areas are less linked to cyberspace, especially because of less accessible website contents. The other main conclusion has to do with the presence of Aveiro and Leiria on the group of the most wired Portuguese localities, despite being middle-sized cities with less than 60 thousand inhabitants, which indicates that the Internet represents an actual challenge to the established order and offers opportunities to reshape urban hierarchies in the context of the knowledge and information society.

The more impressive increase between these two hierarchies is definitively lead by Aveiro, which is the $12^{\text {th }}$ Portuguese city in terms of population, but the $4^{\text {th }}$ in the www.sapo.pt results (and also the $4^{\text {th }}$ in www.google.com), above eight bigger cities: Amadora, Braga, Almada, Coimbra, Funchal, Setúbal, Agualva-Cacém and Queluz. But how can be explained the prominence of Aveiro between the most wired Portuguese cities?

\section{Aveiro Digital City - information content analysis from a case study}

The remarkable prominence of Aveiro on the global networks of information flows can be explained not only by the local economic dynamism or the role of the local University and Research Centres, but also by the successful inclusion of Aveiro in the first Digital Cities public funding programme in Portugal, which started in 1998 and was sponsored by the Portuguese Government and the European Union. 
Figure 5. Portuguese cities with more than 30 thousand inhabitants: opposition between demographic hierarchy and internet presence hierarchy.

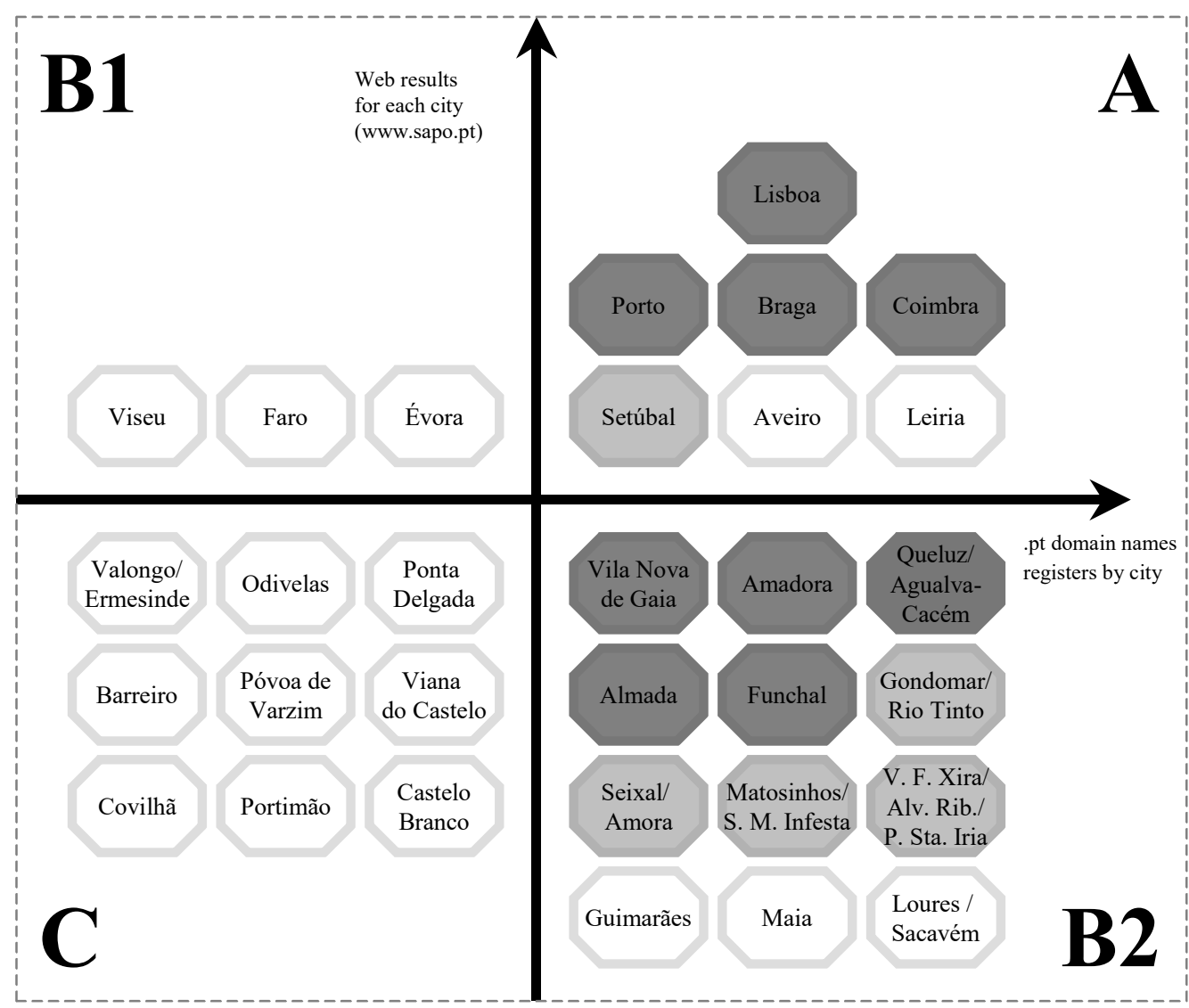

NOTES:

A - Cities with an amount of .pt domain names and web results higher than national averages

B1 - Cities with a No. of web results higher than national average but a No. of .pt domain names lesser than national average

B2 - Cities with a No. of .pt domain names higher than national average but a No. of web results lesser than national average

$\mathrm{C}-$ Cities with an amount of .pt domain names and web results lesser than national averages

The national averages by city are: 162 .pt domain levels; 169 web results for city on www.sapo.pt and 40206 web results for city on www.google.com

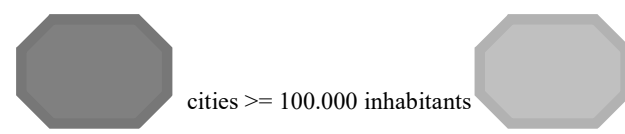

cities $>=60.000$ and

$<100.000$ inhabitants

cities $>=30.000$ and $<60.000$ inhabitants

SOURCES:

Data collected on: Foundation for National Scientific Computing (2003);

www.sapo.pt (accessed between 16 and 28-08-2003);

INE (2002) - Atlas das Cidades de Portugal, Lisboa, Instituto Nacional de Estatística (2001 data for cities population). 
These digital projects (Table 4) comprised diversified initiatives promoted and coordinated by an autonomous organization formed with members of the local government, the local University and the telecommunications operator, PT Telecom, with particular emphasis on infrastructures and digital contents, including local egovernment, e-health, e-business and entertainment (Moutinho and Heitor, 2003).

Table 4. Aveiro Digital City pilot programme (1998-2000)

\begin{tabular}{l|c} 
& $\begin{array}{l}\text { Executed investment by } \\
\text { intervention area }\end{array}$ \\
Interventions Areas & $19 \%$ \\
\hline IA1 - Building a digital community & $20 \%$ \\
IA2 - Local e-government & $16 \%$ \\
IA3 - School and education community services & $4 \%$ \\
IA4 - University community services & $5 \%$ \\
IA5 - Health care services & $4 \%$ \\
IA6 - Social exclusion aid services & $27 \%$ \\
IA7 - Services for commerce and industry & $8 \%$ \\
IA8 - Entertainment, culture and arts & $100 \%$ \\
\hline TOTAL &
\end{tabular}

If we examine the information contents of all 806 web results achieved for Aveiro in www.sapo.pt (Figure 6), we note that some of them are a consequence of the Digital Cities Programme. Results of statistical analyses reveal marked differences between the institutions responsible for such information contents. Half of these websites are provided by economic activities trying to benefit from the opportunities of global economy, especially real estate activities; computer and related activities as well as metallurgy industries (economic activities correspond to the intervention area with more executed investment provided by the Digital Cities public funding programme). The University of Aveiro is also a factor of excellence that distinguishes the city, and it explains why education and training constitute the second most important typology of Aveiro websites. 
Figure 6. Main typologies of the 806 web results obtained for Aveiro city

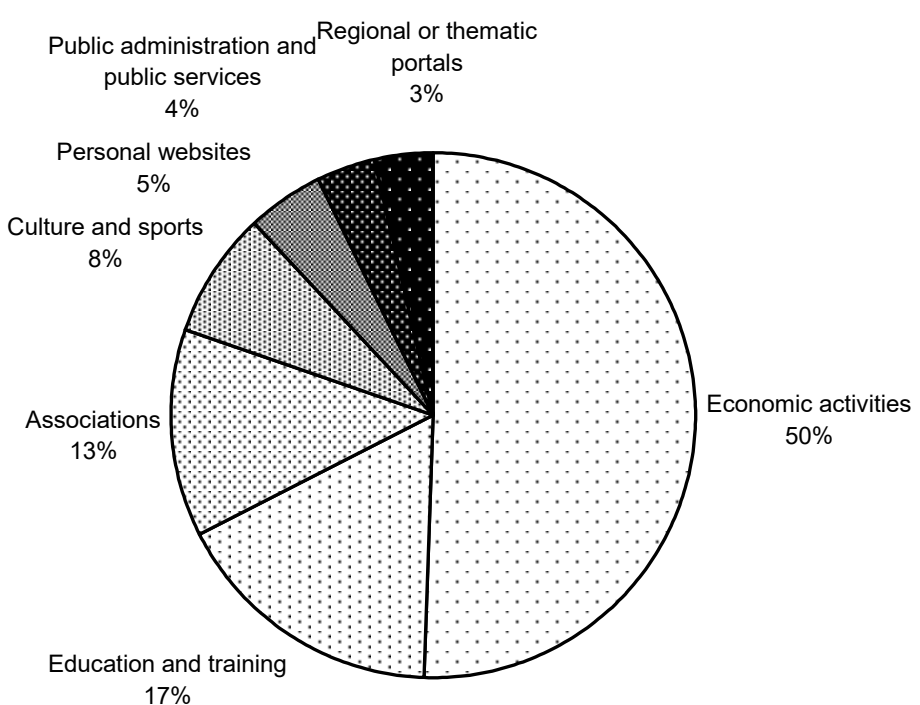

SOURCE: www.sapo.pt (accessed 16-11-2003)

The Portuguese Digital Cities Programme was the first political initiative to develop the information society at local levels, aiming at spreading its benefits throughout Portugal. From all the experiences that were encouraged, Aveiro city soon appeared to have several conditions that made it a political option, providing the opportunity to evaluate concepts and dynamically test new ideas of ICT applications as tools for local development. While others Portuguese digital cities have been developed with specific purposes (for instance: fighting social exclusion, improving economic competitiveness or trying to reduce the disadvantages of inland cities), the Aveiro Digital City has, on the contrary, been developed with a cross-sectional perspective in order to improve living standards and urban life quality in all its dimensions, by the participation of all local agents and the use of ICTs as acceleration tools for a community sustainable development process, in economic, social and cultural aspects .

The main idea consisted in developing a flexible programme, aiming at a progressive and broad mobilization of society with a strong institutional and public participation, with the consequent formalization of new objectives and the constant addition of new projects spontaneously arranged between local agents. With this approach, the idea was 
to achieve an ongoing and adjustable digital city construction, with the continuous development of new solutions for the link between the technological and the sociocultural domains.

Taking into account both the failed and successful experiences that have been characterizing the Aveiro Digital City Programme, we can underline three risks that should be avoided during the implementation of digital cities.

Firstly, favouring the diffusion of ICT infrastructures, as well as the production of ephemeral contents throughout local entities, may be a good way to guarantee good levels of institutional awareness for the benefits of ICT applications. This was one of the most successful goals of Aveiro initiative, but nevertheless, it must be recognised that this decision may have also a negative impact in terms of a small participation of citizens in the information society.

Secondly, presenting and supporting a large variety of practical projects, which for some reason may have to be interrupted (for instance by the lack of financial support or the need to geographically extend the effects of the initiative) is extremely negative, since we are dealing with a difficult purpose - creating new competences and reshape routines. Setting up conditions for long term sustainability of new services provided to citizens is crucial for the success of these kinds of initiatives, which impose systematic and persistent interventions led by human resources with specific skills.

Thirdly, aiming to achieve a consistent and coherent digital city should involve an organizational model with real competences (also in terms of budget allocation negotiations) and a strong supervision, especially in cities such as Aveiro, where we are dealing with a network of local agents developing individual projects and without cooperation habits.

\section{Conclusion}

If the Portuguese cyberspace includes spatial variations and disparities, we believe that measurement, mapping, spatial analysis and interpretation are necessary for a complete understanding of the phenomenon. The main conclusion of this empirical research is that in Portugal the increasing use of Internet seems to increase and reinforce the territorial agglomeration tendencies, instead of acting as equilibrium forces. 
The findings of this study suggest that the role of ICT's in the struggle against traditional spatial inequalities in Portugal is less relevant than expected, since in reality these technologies are strongly affected by the existing spatial structure. The research that we have conducted in order to understand the geographic diffusion of this advanced telecommunications service and its corresponding implications for regional and urban development allow us to contest the theories that emphasize the role of modern means of communication as a way to increase the accessibility to peripheral areas and consequently as a way to split up the effects of centrality. Rather than causing a radical decentralization of population and economic activities, the Internet reinforces the importance of the biggest cities in the Portuguese urban system, especially as regards the metropolitan central cores.

Despite this general trend, the methodological approach used in this study in order to determine the relative position of each city within the space of flows has shown that the magnitude of the existing links between the Portuguese physical space and the global cyberspace indicate a more balanced urban hierarchy, with smaller cleavages between their urban nodes. Moreover, there are some important cities in terms of population density (especially in suburban rings: is the case of Vila Nova de Gaia, Matosinhos and Gondomar in the Porto Metropolitan Area, and Amadora, Almada, Seixal, Queluz and Vila Franca de Xira in the Lisbon Metropolitan Area) that are much less important than some smaller Portuguese cities (like Aveiro) as regards their potential to participate in the organization of the global networks of information flows.

In spite of the Internet activity clearly reinforcing the existing patterns of physical communication rather than creating new ones, and therefore contributing to strengthen territorial disparities, our results seem to suggest that this kind of technological changes has enough potential to generate some adjustments on traditional urban hierarchies, which need to be better analyzed with additional systematic collections of data and further research. 


\section{References}

Anacom, 2004. Serviço de transmissão de dados. Serviço de acesso à internet $-4^{\text {o }}$ trimestre de 2003.

[http://www.anacom.pt/template12.jsp?categoryld=84691] accessed 24-04-2004

Besselaar, P. Van Den, 2004. The life and death of the great Amsterdam Digital City, in: Besselaar, P. Van Den, Koizumi, S. (Eds.), Digital Cities III Information Technologies for Social Capital: a Cross Cultural Perspective, Berlin: Springer-Verlag.

CAD, 2001. Relatório Global da $1^{\text {a }}$ Fase do Programa Aveiro Cidade Digital 19982000, Aveiro: Consórcio Aveiro Digital.

Castells, M., 1992. European cities, the informational society, and the global economy. [http://www.acturban.org/biennial/doc_planners/castells_European_informational_society_glob al_economy.htm] accessed 21-11-2003

Castells, M., 2001. The Internet Galaxy. Reflections on the Internet, Business, and Society, Oxford University Press, Oxford.

Dodge, M., Shiode, N., 2000. Where on Earth is the Internet? An empirical investigation of the geography of internet real estate, in: Wheeler, J., Aoyama, Y., Warf, B. (Eds.), Cities in the Telecommunications Age. The fracturing of geographies, Routledge, London, pp. 42-53.

Drewe, P., 2000. The Internet - how to position capital cities on the European net. [http://web.telecom.cz/iaa/ecaiteda2001.html] accessed 21-11-2003

INE, 2002a. Atlas das Cidades de Portugal, Instituto Nacional de Estatística, Lisboa.

INE, 2002b. Estatísticas do Comércio Internacional 2001, Instituto Nacional de Estatística, Lisboa. 
Moutinho, J., Heitor, M., 2003. Digital Cities and the challenges for a knowledge-based view of the Territory: evidence from Portugal, paper presented at the International Conference Small Firms Strategy for Innovation and Regional Problems, University of Algarve, Faro.

Moss, M., Townsend, A., 2000a. The Internet backbone and the American metropolis, Information Society, 16, 35-47.

Moss, M., Townsend, A., 2000b. The role of the real city in cyberspace: understanding regional variations in internet accessibility, in: Janelle, D., Hodge, D. (Eds.), Information, Place, and Cyberspace: Issues in Accessibility, Springer-Verlag, Berlin, pp. 171-186.

Kitchin, R., 2000. Cyberspace. The World in the Wires, John Wiley \& Sons, Chichester.

Steineke, J. M., 2000. The Web and the cities: explaining spatial patterns of Internet accessibility and use in Norway.

[http://www.rf.no/internet/student.nsf/wvPublNr/2000-116] accessed 21-11-2003

Townsend, A. M., 2000. The Internet and the rise of the new network cities, 1969-1999, Environment and Planning B: Planning and Design, 28, 39-58.

Townsend, A. M., 2001. Network cities and the global structure of the internet, American Behavioral Scientist, 44, 1697-1716.

Zook, M. A., 2001. Old Hierarchies or New Networks of Centrality? - The Global Geography of the Internet Content Market, American Behavioral Scientist, 44, 1679-96.

\section{Notes}

\footnotetext{
1 Castells (1992, p.6) defines space of flows as "the abstract space constituted in the networks of exchange of capital flows, information flows, and decisions that link directional centres among themselves throughout the planet".

${ }^{2}$ The domain name for the websites created with a Portuguese reference.
} 
3 This technique uses the addresses associated with the registration of domain names (such as www.mit.edu) in order to localize the sites of Internet activity in the US.

${ }^{4}$ The Internet Protocol Address is a numeric code used to identify and locate computers on the network. The registry of IP address space lists all the companies and organizations to which allocations have been made. RIPE (Réseaux IP Européens) is the registry entity responsible for covering Europe and surrounding countries.

${ }^{5}$ This results from a limitation of the IP address methodology. "The geographical location at which the IP address space is owned may not necessarily be the same as where the space is actually used, that is, where the Internet-linked computers are located" (Dodge and Shiode, p. 47).

${ }^{6} \mathrm{~A}$ domain name represents a single organisational entity present on the Internet.

${ }^{7}$ The organisational domains may be grouped under CONE domains (.com for commercial businesses, .org for non-profit organisations, .net for networking companies and .edu for educational institutions) or CC (country codes) domains (such as .fr for france, .uk for the United Kingdom or .pt for Portugal). CONE domain names can be registered by anyone regardless of their location but are centrally administrated by a monopoly delegated by the US National Science Foundation (Steineke, 2000). CC domain names are generally administrated by national institutions, which make it more available for this kind of analysis.

${ }^{8}$ FNSC does not have postal addresses of all the registered entities. The registers of pt domain names with this information represent $78 \%$ of the total registers (24724 from a total of 31705 .pt domain names). Unlike the earlier registrations (until 1995-96), nowadays the postal address is a compulsory field during the registration process. 